\title{
In-Situ and Ex-Situ Characterization of Evolution of Defects in AIGaN/GaN HEMTs under Bias
}

\author{
H. Ghassemi ${ }^{1}$, A. Lang ${ }^{1}$, C. Johnson ${ }^{2}$, R. Wang ${ }^{3}$, B. Song ${ }^{3}$, H. Xing ${ }^{3}$, and M. L. Taheri ${ }^{1}$ \\ 1. Department of Materials Science and Engineering, Drexel University, Philadelphia, PA, 19140 \\ 2. Centralized Research Facilities, Drexel University, Philadelphia, PA, 19140 \\ ${ }^{3}$. Department of Electrical Engineering, University of Notre Dame, Notre Dame, IN 46556
}

Nitride semiconductors offer many unique and beneficial properties for a new generation of electronic devices [1]. AlGaN/GaN HEMTs are used in applications where high-power and high-frequency devices are needed. Unfortunately, high-power operating conditions result in unpredictable and catastrophic device degradation [2]. Various techniques have been used to detect and investigate the degradation mechanisms of these devices, including cathodoluminescence spectroscopy, atomic force microscopy, and TEM [3]. However, the formation mechanism of these cracks was not investigated as a function of operating time. As such, quantitative analysis on evolutions of defects and piezoelectric polarization is needed to further understand device failure mechanisms.

The degradation of ex-situ and in-situ biased AlGaN/GaN high electron mobility transistor (HEMT) devices was quantified as a function of defect generation and overall strain evolution in the AlGaN layer using high-resolution transmission electron microscopy (HRTEM) techniques. To study degradation mechanisms as a function of applied bias, in-situ TEM experiments were carried out on cross sections of HEMT devices. In situ studies were coupled with geometric phase analysis to quantify strain in biased devices. In order to observe and characterize the evolution of strain and defects during the operation of a HEMT device, in-situ TEM basing experiments were carried out on lift-out devices. The amount of strain in $\mathrm{AlGaN}$ layer was measured before, during and after the biasing experiment, using HRTEM imaging. Real-time observation of generation of de fects and formation of physical damage provides a fundamental understanding of the unknown reliability of HEMTs under application of bias, which will contribute toward their functionality during application.

In Figure 1a, low-mag scanning electron microscopy (SEM) of a HEMT device is shown and the highermag SEM image in the inset indicates the gate, source and drain electrodes of the device. Dark-field TEM image of a lift-out device is represented in Figure $1 \mathrm{~b}$ which reveals the presence of the threading dislocation in the GaN bulk layer. Figure 1c shows an HRTEM image of an area under the drain side of the gate of a biased device. The corresponding strain map is shown in Figure 1d, in direction parallel to the interface and the strain was measured $+1.67 \%$. Observations of device cross-sections on ex-situ biased devices revealed that the formation of defects mainly on the drain side of the gate. Geometric phase analysis (GPA) of HRTEM images indicated that the tensile strain decreased from $+1.67 \%$ in the unbiased device to $+1.17 \%$ after sufficiently long bias duration. Based on our observations, we propose three different regimes under which a HEMT device undergoes physical degradation during its lifetime. In-situ TEM biasing experiments were also carried out on lift-out devices in order to characterize formation of defects during the application of bias.

In summary, we quantified the evolution of strain and the role of defects under biasing. Based on our results, we propose three different regimes of aging under electrical bias. Changes in sheet polarization charge density supported our ob servations of the defect formation during their lifetime. Our study 
provides a fundamental understanding of the reliability of the HEMTs under application of bias, which will contribute to their increased functionality during application.
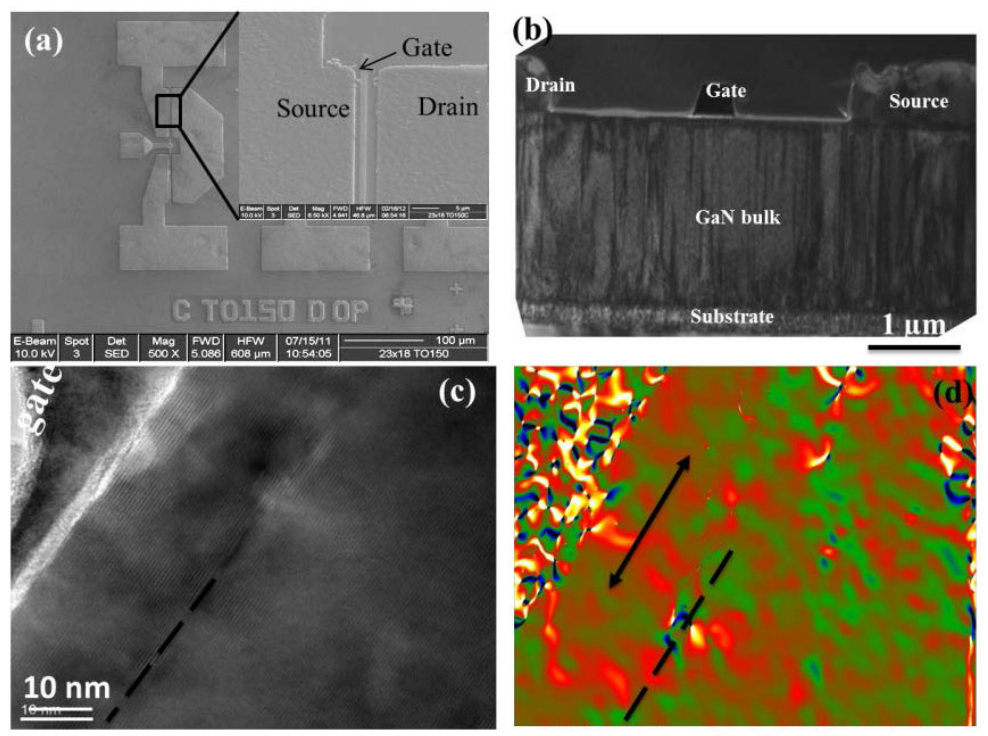

Figure 1 SEM image of a HEMT device. Higher magnification image of the device is shown in the inset to indicate the drain, source and the gate. (b) Dark-field TEM image of the cross section of a lift-out sample. Threading dislocations in the GaN bulk can clearly be observed. (c) HRTEM image of a biased device of the area under the drain side of the gate is shown. AlGaN/GaN interface is indicated with the dotted line. (d) Represents the strain map of the area shown in (c) in direction parallel to the interface, which is indicated with the dotted line.

\section{Reference}

1. Morkoç, H., Handbook of Nitride Semiconductors and Devices, GaN-based Optical and Electronic Devices. 2008: Wiley.

2. Joh, J., et al., Role of stress voltage on structural degradation of GaN high-electron-mobility transistors. Microelectronics Reliability, 2011. 51(2): p. 201-206.

3. Lin, C.-H., et al., Nanoscale mapping of temperature and defect evolution inside operating AlGaN/GaN high electron mobility transistors. Applied Physics Letters, 2009. 95(3): p. 033510. 